\title{
The effect of elastic band assisted gait training to improve the gait kinematics in stroke survivors - a randomized control trial
}

\author{
Priya Sudarshan Visoi, Patitapaban Mohanty* and Monalisa Pattnaik \\ Swami Vivekanand National Institute of Rehabilitation Training and Research, Olatpur, Bairoi, Cuttack, India
}

\begin{abstract}
Introduction: Stroke is the leading cause of serious long-term disabilities, the restoration of walking plays a prime role in his rehabilitation. The reduction in gait performance may prevent the resumption of activities of daily living.

Aim of the study: To compare the effectiveness of elastic band assisted gait training over the task-oriented circuit interval gait training in stroke survivors.

Methodology: A total of 50 male stroke survivors with functional ambulatory category score $\geq 4$, mini mental state $24-30$, normal cardio-pulmonary status and age between 30-60 years were recruited and randomly distributed in two groups. Group 1 received conventional exercises (task-oriented approach in the form of circuit interval gait training) and Group 2 received elastic band assisted gait training with task-oriented approach in the form of circuit interval gait training.

Outcome measures: Stride length (both side), cadence, walking speed and gait profile score (both side). Measurements were taken with gait laboratory before and at the end of 6 weeks of treatment, for 45 minutes divided into nine 5 -minute blocks separated by 5 -minute rest periods 5 days/week.

Results: Overall result of this study showed that experiment group had significantly improved in terms of speed, cadence, stride length of paretic limb, gait profile score of non paretic limb from pre to post test measurements, but no significant improvement in conventional group. Gait profile score of paretic limbs had significantly improved. Both the groups had significantly improved in terms of stride length of non paretic limb from pre to post-test measurements; however, the experiment group showed more improvement than the conventional group.
\end{abstract}

Conclusion: Elastic band assisted gait training over the task-oriented circuit interval gait training is found to be effective for the hemiplegic gait.

\section{Introduction}

Stroke is the leading cause of serious long-term disabilities, including loss of motor, sensory or cognitive function [1]. The restoration of walking plays a prime role in rehabilitation [2]. The reduction in gait performance may prevent the resumption of activities of daily living, which can have an adverse effect on perceived participation [3].

Hemiparetic gait pattern is characterized by being stereotyped with reduced weight bearing on the paretic lower limb. Over activity or shortening of plantar flexor muscles at swing phase giving the equinovarus foot placement [4]. Reduced knee flexion, exaggerated hip circumduction and hip hiking during the swing phase are a common disability in people with stroke [5]. Which movements are energetically demanding and may lead to chronic joint pain $[6,7]$.

Manual assistance during gait training is necessary to properly align the trunk and guide the lower extremity through normal gait trajectory. It is difficult to consistently and adequately control the patient and manually assist dorsiflexion during the swing phase of gait; it can be exhausting and place the physical therapist in a nonergonomic position [8].

There exist devices like Hip flexion orthosis [9], Elastic band orthosis [10], Wearable tubing assistive walking device [11], AFO shaped band [12] and wearable tubing to address ankle dorsiflexion, hip and knee flexion with patient either in non-weight or weight bearing positions. Elastic resistance training has been reported to increase gait, mobility, balance and reduced pain [8-15]. Apart from its use in strengthening program, elastic band can also be used in assisted gait training [15].

\section{Aim of the study}

Our purpose of the study is to compare the efficiency and effectiveness of elastic band assisted gait training over the task-oriented circuit interval gait training to improve the gait kinematics in stroke survivors.

\section{Methodology}

Study design: Randomised Control Trial

Research setting: The study was conducted at the Physiotherapy Department of Swami Vivekanand National Institute of Rehabilitation Training and Research (SVNIRTAR).

${ }^{\star}$ Correspondence to: Patitapaban Mohanty, PhD, Assoc. Prof. (PT), Swami Vivekanand National Institute of Rehabilitation Training and Research, Olatpur, Bairoi, Cuttack, India, E-mail: ppmphysio@rediffmail.com

Received: May 13, 2019; Accepted: June 18, 2019; Published: June 21, 2019 
Inclusion criteria: Patients diagnosed as first episode of stroke with functional ambulatory category (FAC) score $\geq 4$, mini mental state 2430 , normal cardio-pulmonary status and age between 30-60 years of male.

Exclusion criteria: Patient with severe spasticity on Modified Ashworth Scale $(3,4)$, history of any musculoskeletal disorder, other neuromuscular disorder.

Outcome measures: Final Gait analysis was performed in the gait Laboratory which was equipped with 6 infrared cameras recording at $120 \mathrm{~Hz}$ (BTS Smart, BTS Bioengineering, Odisha, India). The system measures kinematic and spatiotemporal parameters based on the Helen Hayes Davis protocol, biomechanical program Tracker and Analyzer (BTS Bioengineering). The stride length of the paretic and non-paretic extremities, velocity, cadence and gait profile score of the paretic and non-paretic extremities were measured.

Subjects/participants: A total of 50 stroke survivors were recruited randomly. Conventional group - 25 subjects and Experimental group 25 subjects.

Procedure: After meeting the inclusion and exclusion criteria through an assessment proforma, informed consent was taken, 50 subjects with mean age 50.22 were taken and divided into two groups namely Experimental group [mean age 51.37] and conventional group [mean age 49.52]. Gait kinematic (Stride length of both side, Cadence, walking speed, Gait profile score of both side) of subjects analyzed with Gait laboratory.

Conventional group-25 subjects [mean age 49.52] received task oriented approach in the form of circuit interval gait training for 45 minutes divided into nine 5 minute blocks separated by 5 minute rest periods, pre-ambulation skill training included supine bridging, reach out in standing with diagonal weight shifts and stepping forward, backward, sideways and up for the first 15 minutes followed by task practice walking upright using manual and verbal cues, heel-toe initial contact, high step marching and then high step, walking with even, increasing step length and/or 2"-3" step width, heel-rises in stance, heel-walking, push-off, appropriate knee flexion in combination with hip extension, exaggerated arm swings, gradually progress to lateral side-steps, longer distances with decreased number of rest intervals and increase velocity in the last 30 minutes.

Experimental group - 25 subjects [mean age 51.37] received elastic band assisted gait training with task oriented approach in the form of circuit interval gait training for 45 minutes divided into nine 5 minute blocks separated by 5 minute rest periods, pre-ambulation skill training included supine bridging, reach out in standing with diagonal weight shifts and stepping forward, backward, sideways and up for the first 15 minutes followed by task practice walking upright using manual and verbal cues, heel-toe initial contact, high step marching and then high step, walking with even steps, increasing step length and/or 2"-3" step width, heel-rises in stance, heel-walking, push-off, appropriate knee flexion in combination with hip extension, exaggerated arm swings, gradually progress to lateral side-steps, longer distances with decreased number of rest intervals and increase velocity in the last 30 minutes.

Treatment was given 5 days a week for 6 consecutive weeks.

\section{Data collection}

Gait kinematic (Stride length of both side, Cadence, walking speed, Gait profile score of both side) of subjects were measured before and at the end of 6 weeks of treatment.

\section{Data analysis}

Statistical analysis was performed using SPSS version 24.0. The Gait parameters [gait profile score, Stride length, Cadence and velocity] were analysed using $2 \times 2$ ANOVA, where there was one between factor [group] with two levels [experimental and control] and one within factor [time] with two levels [Pre and Post]. All pair wise post-hoc comparisons were analysed using a 0.05 level of significance.

\section{Results}

\section{Figure 1: Velocity}

Figure 1 illustrated that there was improvement in walking velocity in both groups following treatment for 6 weeks. The experimental group showed greater improvement as compared to the control group.

There was also main effect for group $\mathrm{F}[1,44,0.05]=0.207, \mathrm{p}=0.652$. There was main effect for time $F[1,44,0.05]=65.808, p=0.000$. The main effects were quantified into timexgroup interaction $\mathrm{F}$ $[1,44,0.05]=28.836, \mathrm{p}=0.00$

Tukey's Post Hoc analysis showed that there was a significant improvement in score for experimental group from pre to posttest measurements but in control group change is not significant. However, both groups are significant different at the end of $6^{\text {th }}$ weeks of intervention.

\section{Figure 2: Cadence}

Figure 2 illustrated that there was improvement in Cadence in both groups following treatment for 6 weeks. The experimental group showed greater improvement as compared to the control group.

There was no main effect for group $\mathrm{F}[1,44,0.05]=1.544, \mathrm{df}=1$, $\mathrm{p}=0.221$. There was main effect for time $\mathrm{F}[1,44,0.05]=11.849, \mathrm{df}=1$, $\mathrm{p}=0.001$. The main effects were qualified into timexgroup interaction $\mathrm{F}$ $[1,44,0.05]=7.000, \mathrm{p}=0.011$.

Tukey's Post Hoc analysis showed that there was a significant improvement in score for experimental group from pre to posttest measurements but in control group change is not significant. However, both groups are significant different at the end of $6^{\text {th }}$ weeks of intervention.

\section{Figure 3: Stride length [non paretic limb]}

Figure 3 illustrated that there was improvement in Stride Length of Non-Paretic Limb in both groups following treatment for 6 weeks. The improvement in stride length was significantly more in the Experimental group as compared to the control group.

There was no main effect for group $\mathrm{F}[1,44,0.05]=0.782, \mathrm{df}=1$, $\mathrm{p}=0.381$. There was main effect for time $\mathrm{F}[1,44,0.05]=61.128, \mathrm{df}=1$,

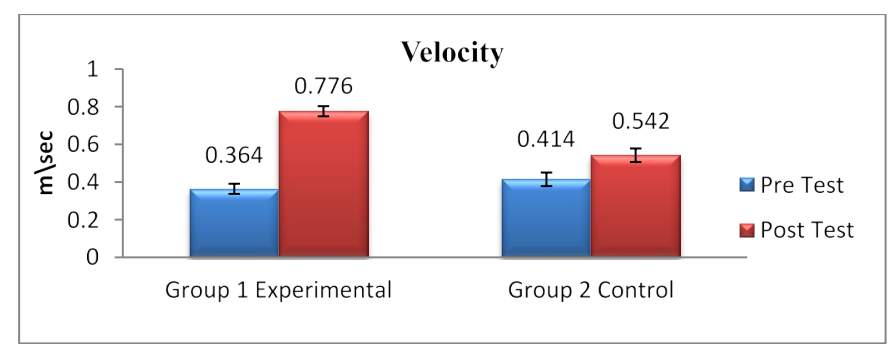

Figure 1. Velocity 


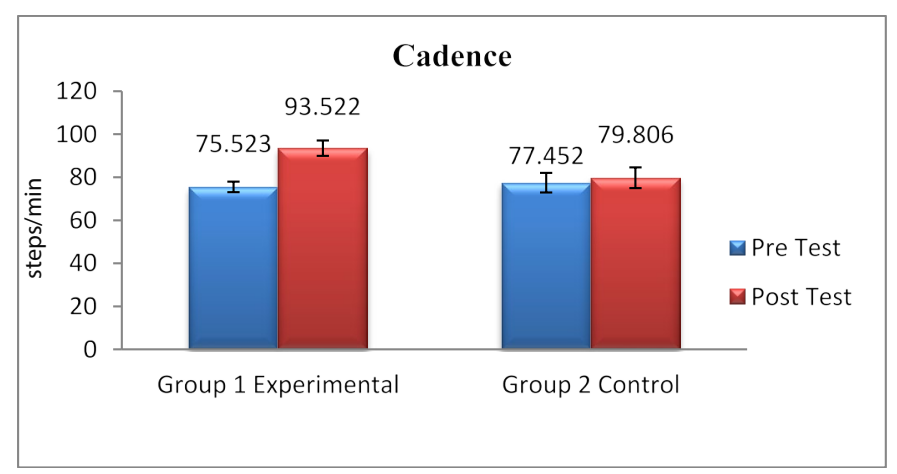

Figure 2. Cadence

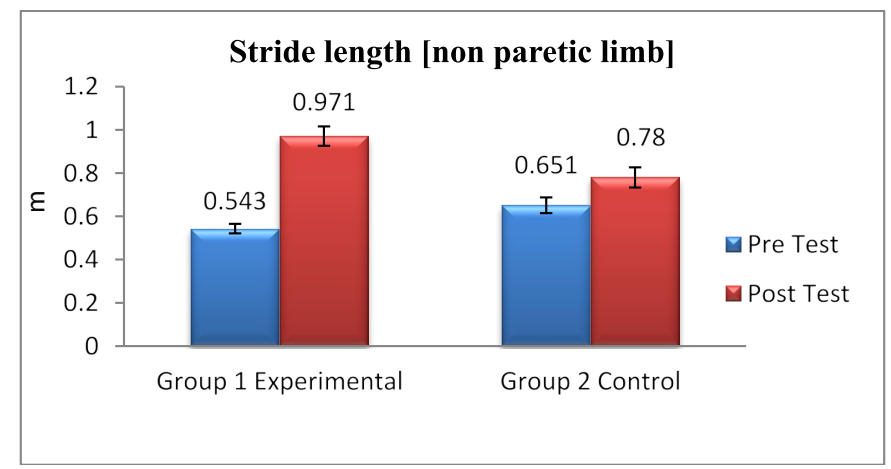

Figure 3. Stride length [non paretic limb]

$\mathrm{p}=0.000$. The main effects were not qualified into timexgroup interaction $\mathrm{F}[1,44,0.05]=19.261, \mathrm{p}=0.000$.

Tukey's Post Hoc analysis showed that there was a significant improvement in score for both groups from pre to post-test measurements. However, both groups are significant different at the end of $6^{\text {th }}$ weeks of intervention.

\section{Figure 4: Stride length [paretic limb]}

Figure 4 illustrated that there was improvement in Stride Length of Paretic Limb in both groups following treatment for 6 weeks. The improvement in stride length was significantly more in the Experimental group as compared to the control group.

There was no main effect for group $\mathrm{F}[1,44,0.05]=0.993, \mathrm{df}=1, \mathrm{p}=$ 0.324 . There was main effect for time $\mathrm{F}[1,44,0.05]=49.270, \mathrm{df}=1, \mathrm{p}=$ 0.000 . The main effects were not qualified into time $\times$ group interaction $\mathrm{F}[1,44,0.05]=15.306, \mathrm{p}=0.000$.

Tukey's Post Hoc analysis showed that there was a significant improvement in score for experimental group from pre to posttest measurements but in control group change is not significant. However, both groups are significant different at the end of $6^{\text {th }}$ weeks of intervention.

\section{Figure 5: Gait profile score [non paretic limb]}

Figure 5 illustrated that there was reduction in Gait Profile Score of non-Paretic Limb in both groups following treatment for 6 weeks. The reduction in score was significantly more in the Experimental group as compared to the control group.

There was no main effect for group $\mathrm{F}[1,44,0.05]=0.224, \mathrm{df}=1$, $\mathrm{p}=0.638$. There was main effect for time $\mathrm{F}[1,44,0.05]=15.823, \mathrm{df}=1$, $\mathrm{p}=0.000$. The main effects were qualified into time $\times$ group interaction $\mathrm{F}[1,44,0.05]=4.377, \mathrm{p}=0.042$.

Tukey's Post Hoc analysis showed that there was a significant improvement in score for experimental group from pre to post-test measurements at the end of $6^{\text {th }}$ weeks of intervention.

\section{Figure 6: Gait profile score [paretic limb]}

Figure 6 illustrated that there was decrease in Gait Profile Score of Paretic Limb in both groups following treatment for 6 weeks. The decrease in score was slightly more in The Experimental group as compared to the control group.

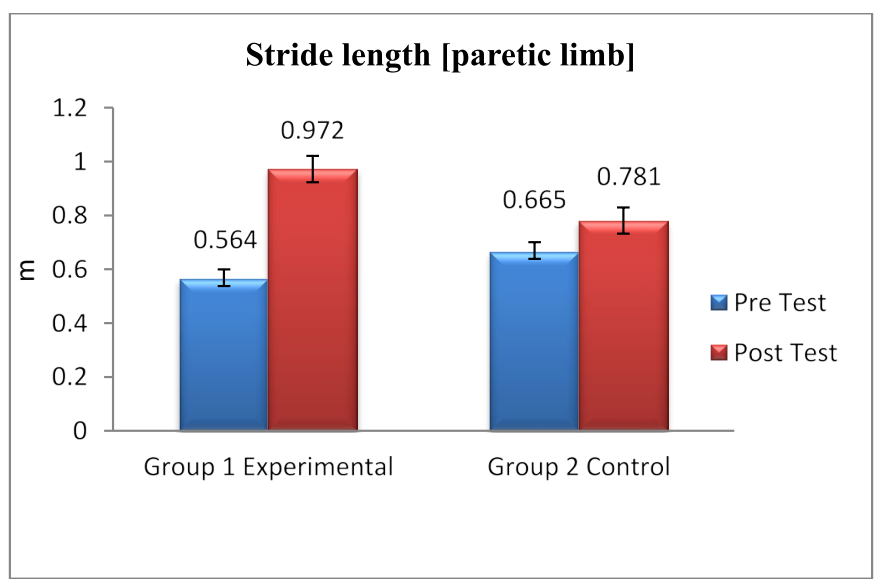

Figure 4. Stride length [paretic limb]

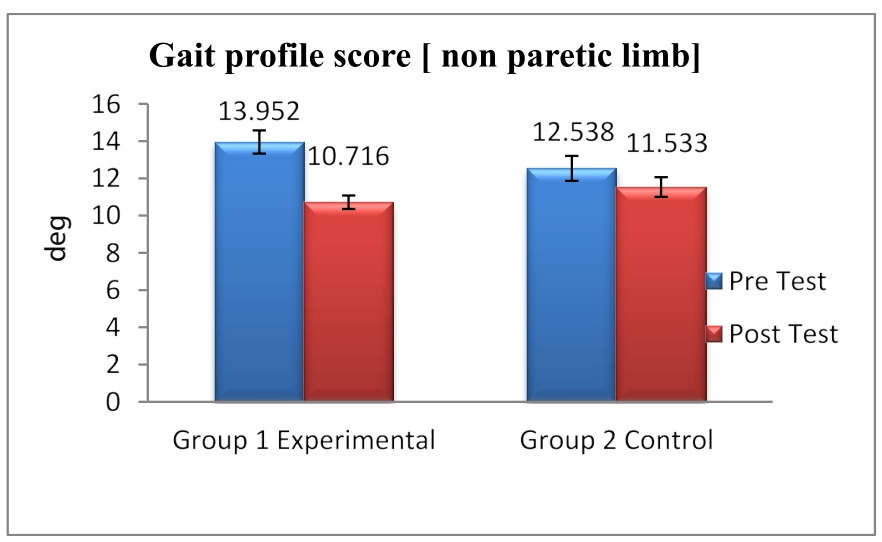

Figure 5. Gait profile score [non paretic limb]

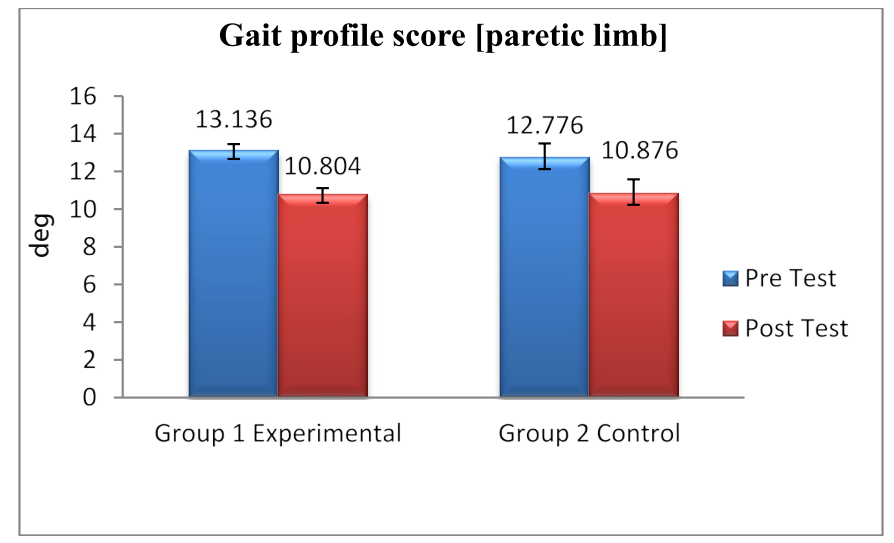

Figure 6. Gait profile score [paretic limb] 
There was no main effect for group $\mathrm{F}[1,44,0.05]=0.051, \mathrm{df}=1, \mathrm{p}=$ 0.823 . There was main effect for time $F[1,44,0.05]=18.317, \mathrm{df}=1, \mathrm{p}=$ 0.000 . The main effects were not qualified into time $\times$ group interaction $\mathrm{F}[1,44,0.05]=0.191, \mathrm{p}=0.823$.

\section{Discussion}

The overall results of this study showed that experiment group had significantly improved in terms of speed, cadence, stride length of paretic limb, gait profile score of non paretic limb from pre to post test measurements, but no significant improvement in conventional group. Gait profile score of paretic limbs had significantly improved from pre to post-test measurements. Both the groups had significantly improved in terms of stride length of non paretic limb from pre to post-test measurements; however, the experiment group showed more improvement than the conventional group in stride length of non paretic limb.

\section{Stride length}

The results of this study suggested that there was a significant improvement in stride length of non paretic limb in both the groups and paretic limb in experimental group from pre to post-test measurements but in conventional group change of paretic limb is not significant at the end of $6^{\text {th }}$ weeks of intervention.

Short steps of non-paretic leg are due to reduced weight shifting toward affected side $[16,17]$. Our subject in conventional group had practice supine bridging, reach out in standing and stepping up, forward, backward, sideways and marching in place which allows sufficient weight-bearing, simultaneously increases stride length on non paretic side.

Supinated foot did not allow proper foot contact and inhibit the smooth propulsions of the both limbs. Decrease in knee flexion momentum during the swing phase reduces stride lengths in paretic limb $[18,19]$.

Similar results were found by Drużbicki et al. who evaluate the effect of gait training on 30 patients with stroke. The program was ten 30-minute training sessions for 2 weeks, effects were evaluated after 6 months, no statistically significant change was observed in step length paretic limb [20].

Our participants in experimental group were capable of achieving longer stride length as elastic band allow proper foot contact during push-off, assist hip pull-off at toe off and longer stride length [21]. An increase in knee flexion momentum through the elastic band shifts the ground reaction force anterior to the lower extremity, thus increasing stride length [22].

We believe that non paretic side has much better mobility then paretic side, which may have contributed to the more improvement in non-paretic side stride length [23].

Similar result was also found by Hwang YI et al. who investigated walking patterns in stroke patient by using the elastic walking band. When the patients walked with the elastic walking band, affected and less-affected stride length were also significantly increased as compared with barefoot walking, more improvement of less affected side. However, we provided feedback, tension selectively for each joint to control the amounts of assistance [13].

Elastic band wrapping around ankle joint functionally act same as an AFO-shaped band which allow ankle stability with mobility. Hwang YI et al. investigated the effectiveness of AFO-shaped band in patients with neurological lesions. Stride lengths on the less-affected side showed significant increases as compared with the barefoot gait suggesting that the stability of the ankle increased during the stance phase. The differences between the stride lengths on the affected side with barefoot and AFO-shaped elastic band were not statistically significant [12].

Elastic band assisted gait training may be equally effective as elastic band orthosis which attached around ankle joint, thereby supporting the hypothesis. Daher $\mathrm{N}$ et al. investigate the effects of elastic band orthosis in stroke patients, which resulted in significantly improved less-affected stride length and no significant difference on the affected side [10]. Elastic band orthosis assists only the ankle joint, whereas in our study use of elastic bands supporting hip, knee and ankle joint was more effective.

The effect of elastic band assisted gait training may be similar to a wearable tubing assistive walking device (WTAWD). It's effects under three conditions (WTAWD, barefoot and conventional elastic band orthosis) was investigated by the Lee SM et al. [11] Stride length on both affected and less affected sides were significantly greater with WTAWD compared to barefoot and conventional elastic band orthosis conditions, thereby supporting the hypothesis.

Veneri D got the similar result with equal stride length bilaterally at 16 weeks which also showed statistically significant improvement similar to our study. Treadmill training with elastic band configured same pattern as our study [15].

The greater improvements in the experimental group compared to conventional group were not only due to the elastic band assistance, but also due to the motivation of subject focusing on gait training and practice and task specific motor activities resulted in cortical reorganisation [24,25].

\section{Cadence}

The results of this study suggested that there was a significant improvement in cadence for experimental group from pre to post-test measurements but in conventional group change is not significant at the end of $6^{\text {th }}$ weeks of intervention.

Stroke survivors walks with slower and smaller steps on non paretic side because of less body weight acceptance on paretic side and failed to produce momentum to push body forward, which reduces the cadence $[16,17]$. Increased apprehension limits the amount of self-initiated training could be contribute to lack of improvement in conventional group [2].

In contrast to our result Wang RY et al. found significantly improved cadence with the 6 tasks of standing in chronic stroke subjects. Task incorporated into our study was different, unlike their which were designed to strengthen the muscles functionally [26].

Our participants in experimental group were capable of achieving greater cadence as elastic band allows proper push-off in the terminal stance and pull off in the pre-swing phase which is the energy generator of ankle plantarflexor and hip flexor respectively and essential for body advancement [27].

Our results were similar to that of Daher N, Lee SM, Hwang YI et al. [10-12].

Though the patient in the conventional group received therapy for same period of time as experimental group, but more improvement was seen in experimental group. The significant difference between the groups reflects the efficacy of gait training with elastic band. 
In our study, we have applied $50 \%$ elongation of elastic band on lateral foot which restrain excessive inversion and 25\% elongation of elastic band on medial foot to maintain in neutral position and prevent sprain by inversion, therefore, we assume that the elastic walking band may reduce this risk, leading to increased walking ability. Elastic band system gait training might subtly have modulated the recruitment patterns, anticipatory adjustment patterns and provided more functional performance when compared to conventional training [21].

\section{Walking speed}

The results of this study suggested that there was a significant improvement in walking speed for experimental group from pre to post-test measurements but in conventional group change is not significant at the end of $6^{\text {th }}$ weeks of intervention.

Increase in gait speed in patients following stroke, is more affected by the Cadence rather than stride length [5]. Subjects of conventional group have not improved in cadence as well as in walking speed.

Lack of long duration and intensive practice actively may be the factor responsible for statistically no significant improvement. In our study, the patients were encouraged to practice by themselves during training but rest of the day their participation rates were low [21].

It would seem that training with longer periods is necessary to improve walking speed which was provided in the study by Salbach $\mathrm{NM}$ et al. this is in contrast with our result, subjects practised similar task-orientated intervention in enhancing walking speed of significant effects. Subjects receive a 6-week intervention 60 minutes per day walking training, including walking on a treadmill additional to functional task included in our study may which was more beneficial [28].

In contrast to our result, more significant improvement in gait speed was observed by Khallaf ME et al. among those who received task specific exercises, gait training, and visual biofeedback for 8 weeks. Whereas our study duration was only 6 week and change were not statistically significant instead of having same protocol [29].

Walking speed depends on the stride length and cadence, our subjects in experimental group has significantly improved in terms of both the factors which could be the reason of improvement. The walking speed thus required a proper push off at ankle, pull off at hip joint and repetitive practice with high motivation results in motor relearning [27].

Similar result was also found by Carda $\mathrm{S}$ et al. patients showed a significant improvement in speed with the use of the HFO [9]. In our study, we assumed additional improvement in speed may be because of elastic band with $25 \%$ elongation passes anteriorly to hip joint instead of lateral and assist in hip pull-off.

The elastic walking band may assist in muscle activation during the swing phase and assisting in ground clearance [30]. It enables the participant to achieve speeds that they cannot attain without ground clearance in swing phase [31].

The observed movement pattern for ankle dorsiflexion and knee flexion in push off phase on the affected limb might be contributed to improvement in gait speed. Limited ankle mobility reduces gait speed [32].

As already mentioned our results were similar to that of Daher N, Lee SM, Hwang YI et al. [10-12].
The tension of the elastic band (25\%) which was crossing posterior thigh was intended to flex the knee joint of the affected lower limb, so that required knee flexion would increase pre-swing knee flexion and speed [33].

Though the patient in the conventional group received therapy for same period of time as experimental group, but more improvement was seen in experimental group. The significant difference between the groups reflects the efficacy of training. Walking speed increases with the stride length and cadence, subjects of experimental group had significantly improved in terms of stride length of both side and cadence but no improvement was there in conventional group neither in stride length of paretic side nor cadence, it could be the reason of improvement in experimental group.

\section{Gait profile score}

The results of this study suggested that there was a significant improvement in gait profile score of non paretic limb in experimental group from pre to post-test measurements but in conventional group change of non paretic limb is not significant at the end of $6^{\text {th }}$ weeks of intervention. Gait profile score of paretic limbs had significantly improved from pre to post-test measurements.

Task-specific exercises tailored with practice of heel-toe initial contact, high step marching and high step walking to the person's interest provide ankle doriflexion, knee flexion and hip flexion repetition and induce a positive long-lasting effect of the task specific exercises [34]. Our study result has pointed out that no improvement was probably due to lack in timing, tuning, and coordinating muscle activation. Circuit interval training with rest of 5 minutes in between without any progression may not increase strength, control, skill, endurance, fitness, and social readjustment no long-lasting cortical reorganisation [35].

Similar result was found by Carda S, Drużbicki M et al. [9,20].

We had wrapped elastic band with $25 \%$ elongation passes anteriority to hip joint assist in hip pull-off which is essential for progression and body advancement in the stance phase, the power produced in hip pulloff is used to achieve lower-limb advancement in the swing phase [27]. We assumed improvement, in our study, may be because of elastic band with $25 \%$ elongation passes anteriorly to hip joint instead of lateral and assist in hip pull-off in better manner.

In double support phase, adequate knee flexion speed at push off is important for normal swing-phase knee flexion [36]. The tension of the elastic band (25\%) which was crossing posterior thigh was intended to flex the knee joint of the affected lower limb, so that required knee flexion was possible during the push-off to swing phases. During pushoff, stored potential energy can be converted to forward momentum that can advance a lower extremity by knee flexion during the initial to mid-swing phases. This forward momentum maintains the knee joint in extension during the mid-swing to initial contact phases. Knee flexion and terminal knee extension bear the weight and absorb shock for stance stability during the initial contact to mid-stance phases [13].

Elastic band wrapping around ankle joint functionally may act same as plantar stop/free dorsiflexion AFO. In our study, Similar to plantar stop/free dorsiflexion AFO, elastic band allow ankle movements in all direction which assist in knee flexion. Mulroy SJ et al. conducted a study to compare the effects of three ankle foot orthosis (AFO) designs on walking after stroke with 30 individuals, were tested in 4 conditions: shoes only, dorsiassist/dorsistop AFO, plantar stop/free dorsiflexion $\mathrm{AFO}$, and rigid AFO. Peak knee flexion in swing was reduced in the 
rigid AFO compared to the plantar stop/free dorsiflexion AFO, but this did not reach statistical significance. The rigid AFO tended to restrict dorsiflexion in stance and knee flexion in swing [32].

Elastic band allows full extension of the metatarso-phalangeal joints and prevents toe clawing, stabilizes the medial longitudinal arch and the $2^{\text {nd }}$ and $3^{\text {rd }}$ rays act as rigid levers capable of withstanding the potentially large bending moments created by the contracting gastrocnemius and soleus muscles [37]. It provides effective manner of "push off" and stored potential energy can be converted to forward momentum that can advance a lower extremity by leading hip, knee flexion, and ankle dorsiflexion during the initial to mid-swing phases [38]. The elastic walking band might help to inhibit tibialis muscle and assist activation of the dorsiflexor through tactile input on the lateral border of the foot to allow ankle dorsiflexion [39].

Though the patient in the conventional group received therapy for same period of time as experimental group, but more improvement was seen in experimental group and reflects the efficacy of training. The results of this study indicate walking training pattern using elastic band system increases the participation because it assists them in a trouble free mobility, initiated the gait pattern which would have resulted in the cortical reorganisation [35]. Taub $\mathrm{E}$ et al. also reported that individuals with stroke achieved symmetrical movement by increasing of their attention and effort [40].

\section{Clinical implications}

The custom-made system was light weight, cost effective, efficient and flexible. In a developing country like India the design would be appropriate. It replaces the need for manual assistance.

\section{Limitations}

No kinetic activities, Cardiorespiratory effects, functional changes and disability contributed to gait were collected, no follow up to know the retention effect of skill, the lack of homogeneity, participants of single geographic location has been taken.

\section{Conclusion}

Stroke survivors showed more improvement in terms of velocity, cadence, stride length of both paretic and nonparetic side and gait profile score of both paretic and nonparetic side when they were subjected to a training protocol which included elastic band assisted gait training with task oriented approach in the form of circuit interval training and preambulation skill training than the task oriented approach in the form of circuit interval training and preambulation skill training alone practiced for a period of 6 week.

\section{References}

1. Tong RK, Ng MF, Li LS (2006) Effectiveness of gait training using an electromechanical Gait trainer, with and without functional electric stimulation in subacute stroke: A randomized controlled trial. Arch Phys Med Rehabil 87: 1298-1304. [Crossref]

2. Davies PM (2000) Steps to follow: The comprehensive treatment of patient with hemiplegia. 2nd edition. Geneve: Springer Verlag.

3. Flansbjer UB, Downham D, Lexell J (2006) Knee muscle strength, gait performance, and perceived participation after stroke. Arch Phys Med Rehabil 87: 974 -980. [Crossref]

4. Bensoussan L, Mesure S, Viton JM, Delarque A (2006) Kinematic and kinetic asymmetries in hemiplegic patients' gait initiation patterns. J Rehabil Med 38: 287294. [Crossref]

5. Perry J, Burnfield JM (2010) Gait analysis: Normal and pathological function. J Sports Sci Med 9: 353.
6. Abdulhadi HM, Kerrigan DC, LaRaia PJ (1996) Contralateral shoe-lift: effect on oxygen cost of walking with an immobilized knee. Arch Phys Med Rehabil 77: 670672. [Crossref]

7. Kerrigan DC, Abdulhadi HM, Ribaudo TA, Della Croce U (1997) Biomechanic effects of a contralateral shoe-lift on walking with an immobilized knee. Arch Phys Med Rehabil 78: 1085-1091. [Crossref]

8. Veneri D (2004) Thera-Band most creative use contest first place winner: Thera-Band assist with swing phase of Gait.

9. Carda S, Invernizzi M, Cognolato G, Piccoli E, Baricich A, et al. (2012) Efficacy of a hip flexion assist orthosis in adults with hemiparesis after stroke. Phys Ther 92: 734739. [Crossref]

10. Daher N, Lee S, Jin Y (2013) Effects of elastic band orthosis (aider) on balance and gait in chronic stroke patients. Phy Ther Rehab Sci 2: 81-86.

11. Lee SM, Cynn HS, Yi CH, Yoon TL, Lee JH (2017) Wearable tubing assistive walking device immediately enhances gait parameters in subjects with stroke: A randomized controlled study. NeuroRehabilitation 40: 99-107. [Crossref]

12. Hwang YI, Yoo WG, An DH, Heo HJ (2013) The effect of an AFO-shaped elastic band on drop-foot gait in patients with central neurological lesions. NeuroRehabilitation 32: 377-383. [Crossref]

13. Hwang YI, Yoo WG, An DH (2013) Effects of the elastic walking band on gait in stroke patients. NeuroRehabilitation 32: 317-322. [Crossref]

14. Hwang YI, Kim KS (2017) Effects of foot pressure using the elastic band with rings during sit-to-stand in persons with stroke. Phys Ther Rehab Sci 6: 159-163.

15. Veneri D (2012) Does combining body weight support treadmill training with TheraBand $^{\circledR}$ improve hemiparetic Gait? J Nov Physiother 2: 114.

16. Patterson KK, Parafianowicz I, Danells CJ, Closson V, Verrier MC, et al. (2008) Gait asymmetry in community-ambulating stroke survivors. Arch Phys Med Rehabil 89: 304-310. [Crossref]

17. Goldie PA, Matyas TA, Evans OM (2001) Gait after stroke: initial deficit and changes in temporal patterns for each gait phase. Arch Phys Med Rehabil 82: 1057-1065.

18. Tyson SF, Thornton HA (2001) The effect of a hinged ankle foot orthosis on hemiplegic gait: objective measures and users' opinions. Clinical Rehabilitation 15: 53-58.

19. Hesse S, Luecke D, Jahnke MT, Mauritz KH (1996) Gait function in spastic hemiparetic patients walking barefoot, with firm shoes, and with ankle-foot orthosis. Int J Rehabil Res 19: 133-141. [Crossref]

20. Drużbicki M, Guzik A, Przysada G, Kwolek A, Brzozowska-Magoń A (2015) Efficacy of gait training using a treadmill with and without visual biofeedback in patients after stroke: A randomized study. J Rehabil Med 47: 419-425. [Crossref]

21. Shumway-Cook A, Woollacott M (2012) Motor control: translating research into clinical practice. $4^{\text {th }}$ edition. Lippincott Williams \& Wilkins. Philadelphia, PA.

22. Van Gestel L, Molenaers G, Huenaerts C (2008) Effect of dynamic orthoses on gait A retrospective control study in children with hemiplegia. Dev Med Child Neurol 50: 63-67.

23. Mojica JA, Nakamura R, Kobayashi T, Handa T, Morohashi I, et al. (1988) Effect of ankle-foot orthosis (AFO) on body sway and walking capacity of hemiparetic stroke patients. Tohoku J Exp Med 156: 395-401. [Crossref]

24. Kleim JA, Jones TA (2008) Principles of experience-dependent neural plasticity: implications for rehabilitation after brain damage. $J$ Speech Lang Hear Res 51: S225-S239. [Crossref]

25. Classen J, Liepert J, Wise SP, Hallett M, Cohen LG (1998) Rapid plasticity of human cortical movement representation induced by practice. J Neurophysiol 79: 1117-1123.

26. Wang RY, Lin PY, Lee CC, Yang YR (2007) Gait and balance performance improvements attributable to ankle-foot orthosis in subjects with hemiparesis. Am J Phys Med Rehabil 86: 556-562.

27. Rose J, Gamble JG (2006) Human walking. 3rd edition. Philadelphia: Lippincott Williams \& Wilkins.

28. Salbach NM, Mayo NE, Higgins J, Ahmed S, Finch LE, et al. (2001) Responsivenes and predictability of gait speed and other disability measures in acute stroke. Arch Phys Med Rehabil 82: 1204-1212.

29. Khallaf ME, Gabr AM, Fayed EE (2014) Effect of task specific exercises, gai training, and visual biofeedback on equino varus gait among individuals with stroke: Randomized controlled study. Neurol Res Int 2014: 693048. 
30. Patil P, Rao S (2011) Effects of Thera-Band® elastic resistance-assisted gait training in stroke patients: A pilot study. Eur J Phys Rehabil Med 47: 427-433.

31. Chen G, Patten C, Kothari DH, Zajac FE (2005) Gait differences between individuals with post-stroke hemiparesis and non-disabled controls at matched speeds. Gait Posture 22: 51-56.

32. Mulroy SJ, Eberly VJ, Gronely JK, Weiss W, Newsam CJ (2010) Effect of AFO design on walking after stroke: impact of ankle plantar flexion contracture. Prosthet Orthot Int 34: 277-292

33. Sulzer JS, Gordon KE, Dhaher YY, Peshkin MA, Patton JL (2010) Preswing knee flexion assistance is coupled with hip abduction in people with stiff-knee gait after stroke. Stroke 41: 1709-1714.

34. Van Peppen RP, Kwakkel G, Wood-Dauphinee S, Hendriks HJ, Van der Wees PJ, et al. (2004) The impact of physical therapy on functional outcomes after stroke: what's the evidence? Clin Rehabil 18: 833-862.
35. Carr JH, Shepherd RB (1987) A motor relearning program for stroke. 2nd edition. Rockville: MD Aspen publishers.

36. Goldberg SR, Anderson FC, Pandy MG, Delp SL (2004b) Muscles that influence knee flexion velocity in double support: implications for stiff-knee gait. J Biomech 37: 11891196. [Crossref]

37. Erdemir A, Hamel AJ, Fauth AR, Piazza SJ, Sharkey NA (2004) Dynamic loading of the plantar aponeurosis in walking. J Bone Joint Surg Am 86: 546-552.

38. Neumann D (2009) Kinesiology of the musculoskeletal system. 2nd edition. United States: Mosby Elsevier.

39. Mojica JA, Nakamura R, Kobayashi T, Handa T (1989) Effect of ankle-foot orthosis (AFO) on body sway and walking capacity of hemiparetic stroke patients. Tohoku $J$ Exp Med 156: 395-401.

40. Taub E, Miller NE, Novack TA, Cook EW 3rd, Fleming WC, et al. (1993) Technique to improve chronic motor deficit after stroke. Arch Phys Med Rehabil 74: 347-354.

Copyright: (C2019 Visoi PS. This is an open-access article distributed under the terms of the Creative Commons Attribution License, which permits unrestricted use, distribution, and reproduction in any medium, provided the original author and source are credited. 